\title{
A Kind of Low Cost Design for Detecting Humidity
}

\author{
Lihua Liu ${ }^{1, a}$ \\ ${ }^{1}$ School of Electrical and Information Engineering, Liaoning Institute of Science and Technology, \\ Benxi, China \\ a benxiliulihua@sina.com
}

Keywords: humidity, MCU, NE555, capacitive humidity sensors, resistive humidity sensor Abstract. The paper introduces the design using capacitive humidity sensors to detect the humidity. By 555 oscillators, the capacitive humidity sensors can change the output frequency. The microchip controller can account the frequency and then according to the math equation, obtaining the humidity information.

\section{Introduction}

Acquiring humidity is very important in some cases, such warehouse or house. If the humidity is so high, the food will be rotted or the mental material will rust. If the humidity is so low, human being will feel dry and unfit even cause fire. So the humidity is important parameters to common living, agriculture or industry.

The typical design for acquiring humidity is as illustrated by Fig.1.

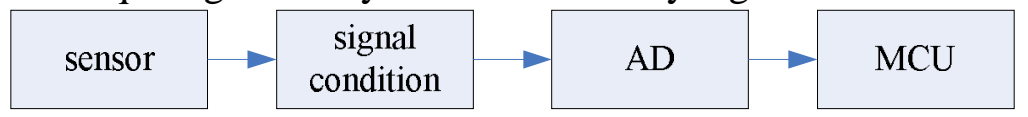

Fig. 1 the typical diagram of humidity circuit

In Fig.1, the humidity sensor transmits the analogy signal, such as voltage or current. The signal condition circuit adjusts the signal to satisfy the requirement. The AD (analogy to digital converter) converts the analogy signal to the digital signal processed by the MCU (Microcontroller Unit).

Now, with rapid technology development, the sensor can integrates more and more circuit on a monolithic chip, which make it easy to employee and reduce the area of PCB(printed circuit board), such DHT11. But we must notice the sensor price will increase obviously. In some price sensitive case, the price will becomes the hamper for future market [1].

In this paper, it introduces a low cost design for the price sensitive occasion.

\section{Design for Humidity Detecting System}

Others than the integrated sensor mentioned above, there are some humidity sensitive sensor with lower price, such capacitive humidity sensor or resistive humidity sensor. Its capacity or resistance can change with humidity.

In Fig.1, it is the capacitive humidity sensor, named HCH-1000. In the Fig.3, it is its characteristic curve. The $\mathrm{X}$-axial means the relative humidity $(\mathrm{RH})$, and its unit is percent. The value on the $\mathrm{X}$-axial is zero means no moisture in the air; and 100 percent means the moisture in the air have reach its limitation.

In Figure 4, it is the resistive humidity sensor and Figure 5 is its characteristic curve. But the y-axial means the resistance value changing with HR.

Sensor. When we carefully observe the two characteristic curves, we will notice the capacitive humidity sensor has the excellent linearity, but the resistive humidity sensor has not yet. The former can give outstanding convenience in the practice application, which could simplify the design. But if consider the price, the former is more expensive than the latter. When the latter applied in the accurate measurement, it needs the compensation by the MCU. 


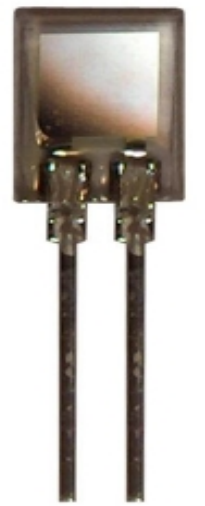

Fig. 2 capacitive humidity sensor

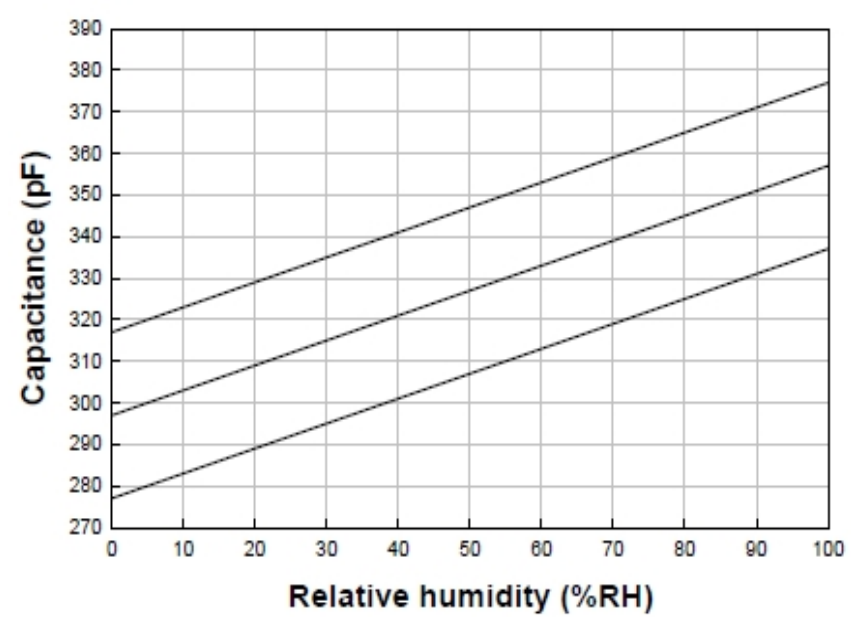

Fig. 3 the characteristic curve

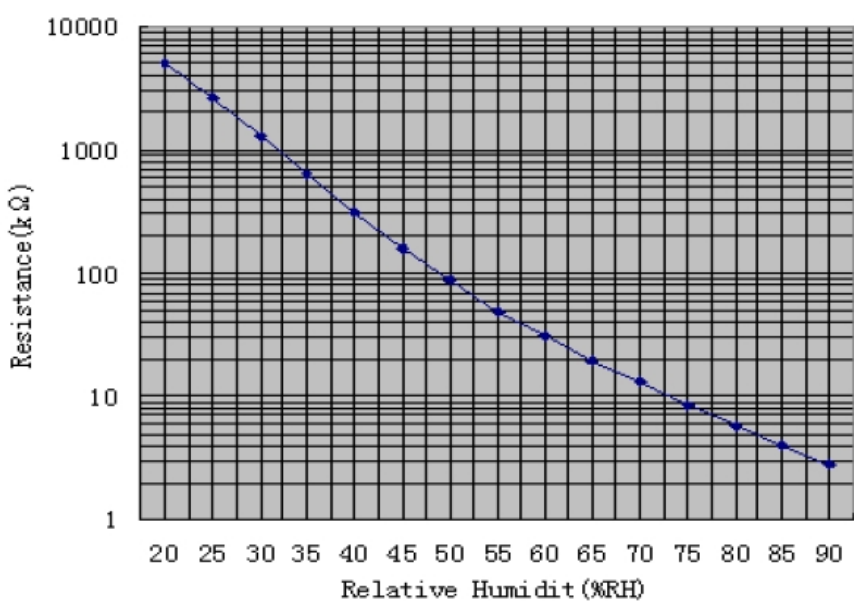

Fig.5 the chrematistics curve
Fig.4 resistive humidity sensor

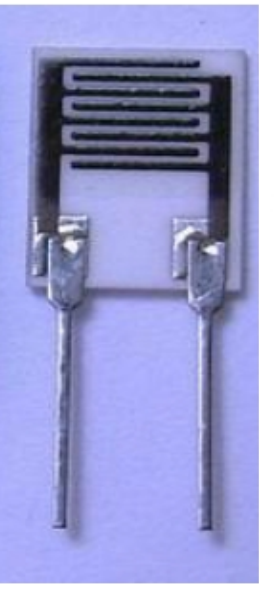

n the application, we need converter the capacity or resistance to the frequency.

Circuit of Frequency Converting. Here we employee the IC chip 555 as the core component to realize the target [2].

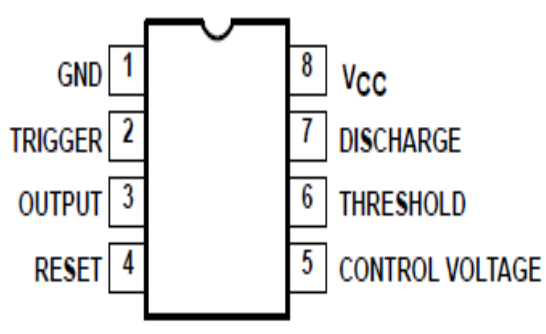

Fig. 6 the pins of NE555

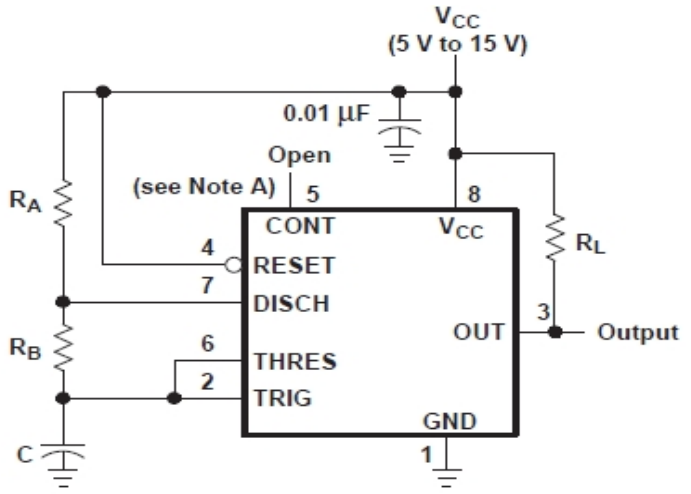

Fig.7 the oscillation circuit of NE555

In the Figure. 6 is the pin out of NE555 IC chip. In the Fig.6 is its circuit for converting the resistance or capacity to the corresponding frequency. The output frequency equation can be described as following:

$$
f=\frac{1}{0.7(R a+2 R b) C}
$$

In above formula, the $\mathrm{Ra}, \mathrm{Rb}, \mathrm{C}$ is in the Fig.7. When convert the capacity to the frequency, the capacitive humidity sensor can replace the capacity $\mathrm{C}$ in the Fig.6. When convert the resistance to the frequency, we can replace the $\mathrm{Rb}$ with the resistive humidity sensor $[4,5,6]$. 
The simulation can demonstrate the design idea in the Fig.8 and Fig.9.

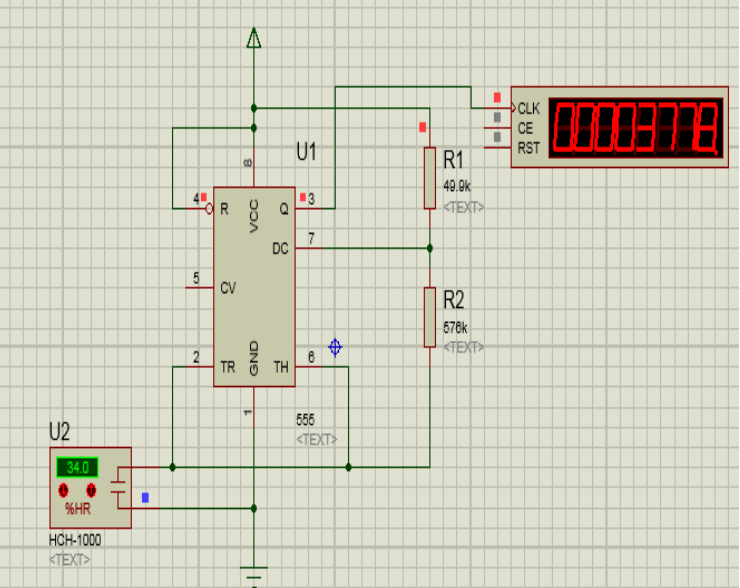

Fig. 8 the simulation part 1

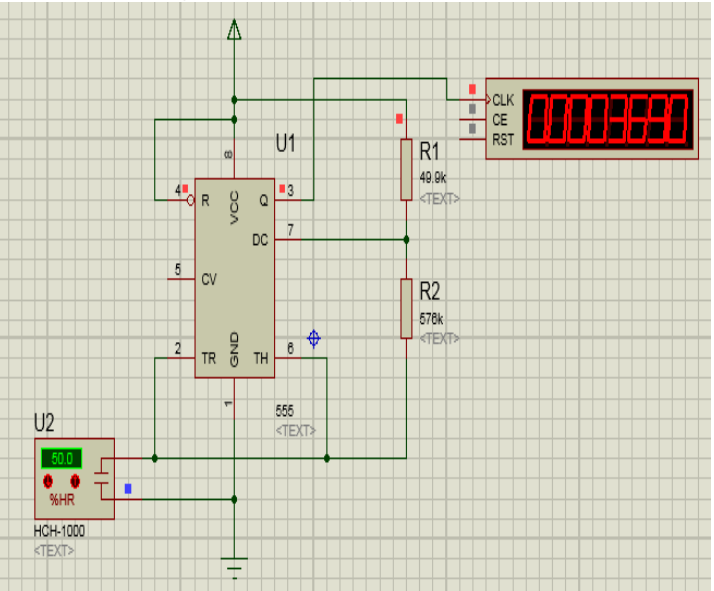

Fig.9 the simulation part 2

In the design, we can take $\mathrm{Ra}$ as $49.9 \mathrm{~K}, \mathrm{Rb}$ as $576 \mathrm{~K}$, and the simulation result in the Fig.8 and Fig.9 shows the output frequency varies with capacitive humidity sensor. From Eq.1, we know the output cycle is proportional to the $\mathrm{C}$, which is so suitable for the capacitive humidity sensor $\mathrm{HCH}-1000$.

Interface with MCU. The MCU reads the output frequency and then calculate the HR value. The hardware can realize by the following protel schematics, Fig.10.

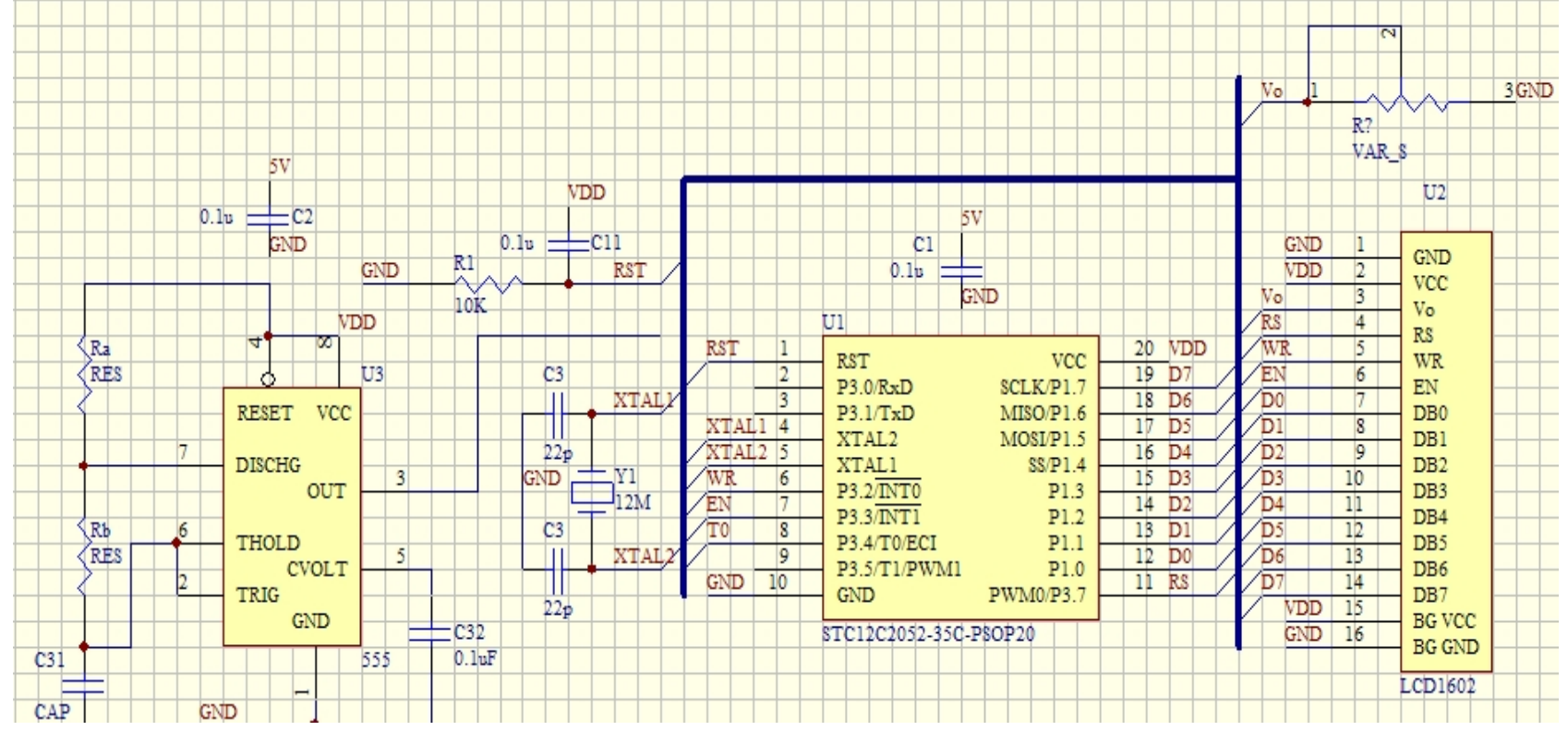

Fig.10 the hardware design

In the design, considering the price, we choose the STC12C2051, which is domestic MCU and has very low price. It receives the frequency of NE555 through the T0 input pin, and then calculates the frequency. According to Eq.1, we know the real capacity or resistance value, and converts it to the HR. In order to improve the accuracy, I choose the $12 \mathrm{Mhz}$ crystal value [7].

Software Flow Chart and Program. Software program lies in the MCU Rom (Read only Memory), which manipulates the MCU. In the design, the software is compiled in the Keil C environment.

The display component is LCD1602, which has low price comparing to the other display component.

The software finishes the LCD1602 initiation, and ready to show the relative information on it. When the LCD1602 initiation is successful, the MCU begins to read the frequency. In the course, the MCU setups timer0, and accumulates the total clock rising times in one second. After it, the MCU calculates the cycle, then has the HR value according to Equ.1 and displays it on the LCD1602. If employee the resistive humidity sensor, the software must first store the calculation data in the Rom, then read it and calculate the accurate HR value. 
The software flow chart is described as below in Fig.11:

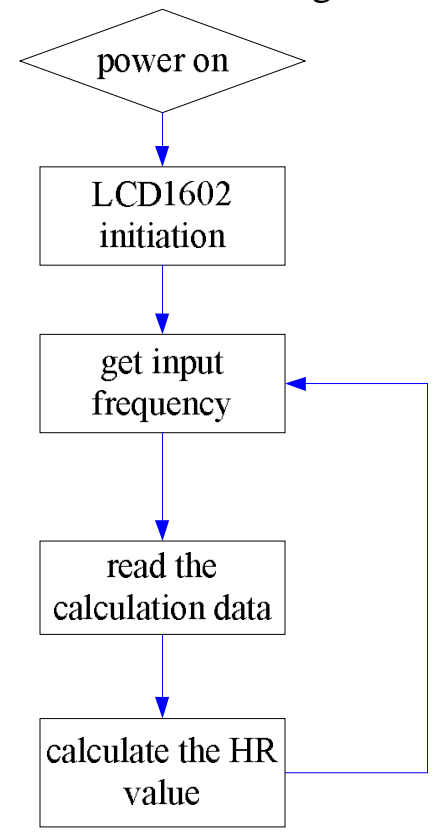

Fig.11 software chart

\section{Conclusion}

By using of capacitive humidity sensor and resistive humidity sensor, we can realize the humidity detecting with lower price.

We also accomplish the hardware and software design. The resistive humidity sensor has the cheapest price, but it needs the complicated compensation by the MCU, so it is most suitable for the two bits switch control condition.

The capacitive humidity sensor has the excellent linearity, so it has the highest ratio between the function and price.

\section{References}

[1] X. Bai, D. L. Guan, R. Chen, "Detect Humidity and Temperature of Multiple Points by CAN Bus", Advanced Materials Research, Vols. 805-806, pp. 1007-1010, Sep. 2013

[2] Information on http://www.TI.com.cn

[3] Wenjie Liu, Maoqin Zhang and Xiaoyu Zhou: Consumer Electronics, Communications and Networks (CECNet), 2011 International Conference on, (Xianning, China, April 16-18, 2011), p.1819

[4] Xiaohong Yang, Yaoliang Xu, Ning Yang, Lei Xia and Wanjian Zhao: Information Science and Engineering (ISISE), 2010 International Symposium on, (Shanghai,China,December 24-26,2010), p.11

[5] Chang, D., Ming Jieh Cheng, Shun-Yu Chan and Jen-Hao Teng: TENCON 2006 IEEE Region 10 Conference, (Hongkong,China,November 14-17, 2006), p.1

[6] Tingyun Chen, Yimin Yang, Song Zhang and Zhichang Luo, Intelligent Information Technology Application, Workshop on , (Zhang jiajie,China,December 2-3,2007), p107-111

[7] Wubing Tang and Jiping Xiu: Techniques of Automation and Applications, Vol.26(2007), p. 118-121 (In Chinese) 